\title{
La competencia transversal "Comprensión e Integración”. Metodologías aplicadas para su implementación en los estudios universitarios del Grado en Gestión y Administración Pública
}

\author{
Marín-Sánchez, María del Mar ${ }^{a}$; Osorio-Acosta, Estefania ${ }^{b}$; Sosa-Espinosa, \\ Asenet $^{c}$; Tolosa-Robledo, $M^{a}$ Luisa ${ }^{d}$ \\ ${ }^{a}$ CEGEA, Departamento de Economía y Ciencias Sociales, Universitat Politècnica de València, España, \\ mmarins@esp.upv. ${ }^{b}$ INECO, Departamento de Economía y Ciencias Sociales, Universitat Politècnica de València, \\ España,. esosac@esp.upv.es 'Departamento de Urbanismo, Universitat Politècnica de València, España, \\ assoes@urb.upv.es. ${ }^{\mathrm{d} D e p a r t a m e n t o ~ d e ~ C o m u n i c a c i o ́ n, ~ U n i v e r s i t a t ~ P o l i t e ̀ c n i c a ~ d e ~ V a l e ̀ n c i a, ~ E s p a n ̃ a . ~}$
}

\begin{abstract}
In this paper we analyze the implementation of the Transversal Competence "Understanding and integration" through the subjects of Management and Public Administration Studies. It is kept in mind that the proficiency level of the competence can not be the same for 1st and 2nd years, which would correspond to competence level 1, that for 3rd and 4th years, to which they correlate a higher level 2. It is concluded that the methodologies used are very different, so it can be inferred that the overall evaluation is correct.
\end{abstract}

Keywords: transversal competences, comprehension and integration, competency training, competence assesment.

\section{Resumen}

A través del presente trabajo se analiza la implementación de la Competencia Transversal "Comprensión e integración" en las asignaturas de los estudios de Gestión y Administración Pública en las que se está evaluando. Se tiene presente que el nivel de dominio de la competencia no puede ser el mismo para los alumnos de $1^{\circ}$ y $2^{\circ}$, a los que correspondería el nivel 1 de la competencia, que para los alumnos de $3^{\circ}$ y $4^{\circ}$ a los que correponde un nivel superior 2. Se concluye que las metodologías empleadas son muy diferentes por lo que se puede inferir que la evaluación global es correcta.

Palabras clave: competencias transversales, comprensión e integración, formación en competencias, evaluación de competencias. 


\section{Introducción y Objetivos}

Las denominadas Competencias Transversales (Gonczy, 2001) tienen un indudable valor en el desempeño profesional de los titulados universitarios. Así, la UNESCO, en la Conferencia Mundial sobre Educación Superior de París de 1998, señaló como uno de los ejes prioritarios la formación en competencias (Arroyo-Cañada, 2019), por lo que cada vez son mas las Universidades que consideran prioritario la evaluación por competencias. En este sentido la Universitat Politècnica de València (UPV) ha puesto en marcha un Proyecto institucional para incluir de forma explícita la exigencia de que nuestros estudiantes se formen en estas competencias y sean evaluados para poder ser acreditado su nivel de dominio.

El objetivo del presente trabajo es analizar como se ha implementado la Competencia transversal de Comprensión e Integración de los conocimientos y las enseñanzas, en el caso de los estudios del Grado en Gestión y Administración Pública de la UPV, y así poder concluir si realmente los alumnus consiguen el nivel de dominio de la competencia.

\section{El Proyecto Institucional de Competencias Transversales de la Universitat Politècncia de València. La Competencia Transversal "Comprensión e Integración}

El Proyecto Institucional de la UPV ${ }^{1}$ tiene como objetivo fundamental:

- Establecer la estrategia de evaluación sistemática de las Competencias Transversales, definiendo como se adquieren y como deben ser evaluadas, y

- Acreditar la adquisición de dichas competencias.

Con este Proyecto institucional se puede afirmar que la UPV podrá:

- Proporcionar a sus egresados un valor añadido que puede diferenciarlos de otros egresados y, en consecuencia, hacer más atractivos los estudios ofertados frente a ofertas similares de otras universidades.

- Poner en valor la capacitación de nuestros egresados de cara a los empleadores.

- Explicitar la adquisición de las competencias de cara a acreditaciones internacionales.

Así se han definido trece Competencias Transversales, siendo la primera de ellas la que nos ocupa en este trabajo de coordinación: "Comprensión e Integración". Comprender quiere decir "percibir y tener una idea clara del que se llama, de lo que se dice, se hace o sucede, o descubrir el sentido profundo de algo" e Integrar implica "completar un todo con las partes que faltaban o fusionar dos o más conceptos divergentes en una síntesis". Para demostrar que algo se ha comprendido, la persona identifica y recupera la información, y lo explica con sus palabras, interpretando e integrando las ideas desde su perspectiva.

${ }^{1}$ Denominado Proyecto de Competencias Transversales (2015). 
Los tres niveles de dominio que se contemplan son:

Nivel 1: Describir, relacionar e interpretar situaciones y planteamientos sencillos. Se correspondería con asignaturas de $1^{\circ}$ y $2^{\circ}$ de Grado.

Indicadores:

- Identificar correctamente las ideas o conceptos de un texto, problema o ejercicio.

- Explicar correctamente, con sus propias palabras, las ideas planteadas en un texto, problema o ejercicio.

- Interpretar, expresando de manera personal, las ideas o conceptos planteados en un texto, problema o ejercicio.

- Integrar diferentes ideas o conceptos de la asignatura en sus análisis.

Nivel 2: Proceder con lógica y relacionar e integrar conceptos en situaciones complejas. Se correspondería con asignaturas de $3^{\circ}$ y $4^{\circ}$ de Grado

Indicadores:

- Clasificar y establecer relaciones significativas entre diferentes ideas o conceptos.

- Proporcionar sentido a los planteamientos que se le presentan para poder interpretarlos.

- Diferenciar los factores causantes o consecuentes de un hecho.

- Transferir los contenidos a la práctica, integrándolos en un contexto.

- Integrar elementos de diferentes asignaturas o áreas en sus análisis de la realidad.

Nivel 3: Identificar las carencias de información y utilizar el conocimientos con un enfoque globalizador.

Indicadores:

- Identificar lagunas de información o falta de coherencia en las argumentaciones.

- Establecer generalizaciones o relaciones causa-efecto.

- Expresar sus ideas y generar conclusiones, partiendo de diferentes datos y sus relaciones.

- Mostrar una visión sistémica entre varias disciplinas y/o áreas de conocimiento.

Las actividades formativas para el desarrollo de esta competencia implican interpretar información de forma reflexiva y precisa para lo cual se requiere entre otras las siguientes actividades:

- Comparar conceptos, principios y teorías, examinar parecidos y diferencias entre dos o más objetos, ideas, situaciones, etc.

- Clasificar: agrupar objetos, sucesos, ideas, etc. según sus características comunes precisando el mejor criterio de agrupación, con el fin de organizar eficazmente el conocimiento y las actividades profesionales y de la vida diaria, etc.

- Analizar y sintetizar. El análisis consiste en aislar los elementos constituyentes del todo, identificándolos, relacionándolos entre sí y con el todo. La síntesis supone 
la integración de todas y algunas partes para producir alguna nueva configuración significativa, alguna conclusión, etc.

- Secuenciar: consiste en ordenar series de elementos que se suceden unos a otros según un criterio que marca la dirección de la progresión.

- Descubrir razones: consiste en indagar las razones ciertas, dudosas e inciertas que sustentan un argumento, una conducta, una información, etc. Evaluando si las mismas apoyan y justifican el que se desea comunicar y también su solidez, relevancia y suficiencia.

Este tipo de actividades se pueden desarrollar siguiendo metodologías de diferente tipo, como por ejemplo:

- Actividades grupales

- Exposiciones orales

- Foros y debates

- Lecturas

- Prácticas de laboratorio

- Preguntas

- Problemas

- Proyectos

- Redacción de informes

- Seminario

Entre los posibles procedimientos de evaluación de esta competencia, destacamos:

- One minute paper

- Sondeo

- Pruebas objetivas

- Redacción de informes

- Autoevaluación

- Evaluación entre iguales

- Elaboración de mapas conceptuales

\section{Metodologías aplicadas en las asignaturas que son punto de control en la Competencia transversal Comprensión e Integración}

En el Grado de Gestión y Administración Pública son 5 las asignaturas en las que se evalúa especialemnte la competencia transversal de "Comprensión e Integración", como se muestra en la Tabla 1. 
Tabla 1. Asignaturas en las que se evalua la CT “Comprensión e Integración”

\begin{tabular}{ll}
\hline \multicolumn{1}{c}{ Nivel 1} & \multicolumn{1}{c}{ Nivel 2} \\
\hline Contabilidad Pública $\left(2^{\circ}\right.$ curso $)$ & Gestión Presupuestaria $\left(3^{\circ}\right.$ curso $)$ \\
$\begin{array}{l}\text { Información y Documentación } \\
\text { Administrativa }\left(2^{\circ} \text { curso }\right)\end{array}$ & Gestiòn tributaria $\left(3^{\circ}\right.$ curso $)$ \\
& \\
& Politicas Públicas y técnicas de \\
& Participación Ciudadana (4 curso)
\end{tabular}

Fuente: elaboración propia.

\subsection{Asignatura de "Información y Documentación Administrativa"}

Es una asignatura obligatoria de $2^{\circ}$ curso, por lo que correspondería al Nivel 1 de dominio en la competencia.

Sobre cada tema se plantea una actividad que el alumno va realizando. Asimismo realiza un examen sobre la actividad con preguntas de respuesta múltiple. El alumno va configurando un portfolio en el que incluye la actividad, el examen y un Glosario. Este portfolio se entrega a final de curso y es corregido. También se asigna a cada estudiante una institución valenciana pública para que el alumno, con ayuda de la bibliografía, cree una instancia para los usuarios de esa institución. La nota de la asignatura es también el grado en que se alcanza la Competencia. En resumen, en esta asignatura se realiza:

- Actividad formativa: actividades grupales; preguntas, prácticas de laboratorio (descritas en el primer párrafo).

- Procedimiento de evaluación: portfolio, observación, redacción de informe escrito.

- Instrumento de evaluación: Apartir de la nota obtenida se le asigna una letra cuya equivalencia con las notas se muestra acontinuación, de modo que la "Excelencia" sería a la letra A, "Adecuado" se define con la letra B, "En Progreso" corresponde a la letra C y "Sin Alcanzar" sería la letra D. Definiendo como criterio, a partir de la nota obtenida en la asignatura, el siguiente modo:

- $\quad$ D: $<2,5$ puntos

- C: $2,5-4,9$ puntos

- B: $5,00-7,5$

- $\mathrm{A}:>7,5$

\subsection{Asignatura "Políticas Públicas y Técnicas de Participación Ciudadana"}

Es una asignatura OPTATIVA de $4^{\circ}$, por tanto del nivel 2 de dominio de la competencia.

En la misma se realizan 3 prácticas de aula, y un trabajo académico. A través de los mismos se estudian y analizan contenidos propios de la materia, de forma que el alumno integra lo aprendido. La nota media en dichas actividades sirve para la calificación de la 
competencia transversal, pues a través de la misma se demuestra su compresión. En resumen:

- Actividad formativa: prácticas de aula en las que el trabajo se desarrolla a través de actividades grupales, y trabajo académico también en grupo

- Procedimiento de evaluación: observación del trabajo desarrollado en el aula dia a dia, y evaluación del informe escrito que se deriva tanto de las practicas como del trabajo académico.

- Instrumento de evaluación: escala de observación cualitativa en la que finalmente la "Excelencia" sería a la letra A, "Adecuado" se define con la letra B, "En Progreso" corresponde a la letra C y "Sin Alcanzar" sería la letra D.:

- D: 0,00-4,00 puntos

- C: 4,01-6,60 puntos

- B: $6,61-8,60$

- A: $8,61-10,00$

Adicionalmente, en esta asignatura, la detección de plagio en los trabajos académicos supone la evaluación negativa de la competencia transversal.

\subsection{Asignatura de "Gestión Presupuestaria"}

Esta asignatura es obligatoria de $3^{\circ}$ curso y se mide el nivel 2 de dominio de la CT. Se realiza un trabajo en grupo, máximo 3 personas, que elabora un informe, combinando trabajo autónomo con tiempo presencial de aula. Deben elegir el Presupuesto de una Institución y analizarlo en profundidad, comprobando que cumple la normativa y siguiendo para ello la dirección del profesor. Los miembros del equipo deberán exponer oralmente el informe elaborado. Se evalúa al grupo mediante el trabajo escrito y también de forma individual a cada estudiante, mediante rúbrica, atendiendo al formato de la presentación, la claridad expositiva, la integración de conocimientos y la profundidad de los argumentos empleados. La duración estimada es de 3-4 semanas a partir del primer examen parcial de la asignatura. En resumen:

- Actividad formativa previa: actividades grupales; prácticas de aula

- Procedimiento de evaluación: observación del trabajo realizado día a día, evaluación entre iguales; evaluación del informe escrito así como de la presentación.

- Instrumento de evaluación: rúbrica, escala de observación. La equivalencia empleada con la nota númerica supone que finalmente la "Excelencia" sería a la letra A, "Adecuado" se define con la letra B, "En Progreso" corresponde a la letra C y "Sin Alcanzar" sería la letra D.:

- $\quad$ D: $<4,5$ puntos

- C: 4,5-5,4 puntos

- B: $5,5-7,5$

- $\mathrm{A}:>7,6$ 


\subsection{Asignatura de "Gestión Tributaria"}

Es obligatoria de $3^{\circ}$ curso, por tanto de nivel 2 en el dominio de la competencia.

Se realizan 4 prácticas de laboratorio en las que se resuelve un caso con ayuda del Programa Informático de ayuda de la Agencia Tributaria española en el que los alumnos pueden revisar el modelo oficial de presentación del Impuesto y el cual deben completar con la solución del caso. De ese modo integran los conocimientos adquiridos al tener que decidir cual es la mejor manera de calcular la liquidación del impuesto. La evaluación del correcto desempeño se realiza a través de un examen tipo test on line empleando la herramienta de la intranet que utilizamos, Poliformat. La nota media en dichas prácticas sirve para la calificación de la competencia transversal.

- Actividad formativa: Prácticas de laboratorio

- Procedimiento de evaluación: Pruebas de respuesta corta y Pruebas de ejecución.

- Instrumento de evaluación: Notas obtenidas en las pruebas de respuesta corta. A partir de esta nota obtenida en las prácticas de laboratorio, se realiza su correspondencia con letras donde la "Excelencia" sería a la letra A, "Adecuado" se define con la letra B, "En Progreso" corresponde a la letra C y "Sin Alcanzar" sería la letra C.del siguiente modo:

- $\quad$ D: $<2,5$ puntos

- C: $2,5-4,9$ puntos

- B: $5,00-7,5$

- $\mathrm{A}:>7,5$

\section{Ventajas e Inconvenientes observados en el análisis comparado}

La principal ventaja que se observa es la diversidad y variedad en las actividades formativas empleadas en cada asignatura para evaluar la competencia transversal. Cabe destacar que las Prácticas de aula implican la resolución de un caso práctico en ocasiones en equipo, o de forma individual, o bien mediante entregas por escrito. En cambio en las prácticas de laboratorio se emplean herramientas informáticas, o bien se le obliga al alumno a consultar textos legales, impresos o modelos tribuatarios para que la resolución del caso que se plantea sea próximo a la realidad. Esto es muy positivo porque implica que la evaluación de la Competencia es efectiva, dado que se analizan los resultados del aprendizaje del alumno con distintas herramientas y así se asegura su consecución por parte del estudiante.

Asimismo los procedimientos de evaluación son también distintos, adecuándose a las actividades formativas realizadas en cada asignatura, lo cual entendemos redunda en la calidad docente finalmente alcanzada. En algunas actividades se ha empleado la rúbrica, en otras la observación evaluando la participación y el desempeño de cada estudiante, en otras exámenes de respuesta corta, otras elaboración de informes y portfolios, etc.. 
A pesar de la viariedad tanto metodológica como en formas de evaluación, estas presentan algo en común, ya que todas ellas se inscriben en actividades prácticas, que implican la búsqueda de soluciones a distintas situaciones muy próximas a la realidad. Esto refleja el posicionamiento de los docentes hacia la potenciación de un rol activo en el proceso de enseñanza-aprendizaje del alumnado, próximo a la metdología de aprender haciendo "learning by doing", adecuado en el desarrollo del pensamiento comprensivo".

En lo que se refiere a los inconvenientes detectados se señala que la gran cantidad de profesorado asociado (un 52\% de los profesores que imparten su docencia en los estudios de GAP son Asociados a tiempo parcial), dificulta la correcta implementación de las distintas actividades programadas que aseguren que su ejecución es igual en todos los grupos, generándose a veces diferencias difíciles de superar.

Sería deseable incrementar el nivel de coordinación en las asignaturas que deben formar al estudiante en la misma Competencia Transversal, y especialmente si deben evaluarla.

Asimismo, otros inconvenientes pero de índole material se refieren a los recursos de infraestructuras dado que no se pueden emplear laboratorios informáticos en algunas actividades que incrementaría su eficiencia notablemente.

Tabla 2. Ventajas e Inconvenientes. Análisis comparado. CT “Comprensión e Integración"

\section{Ventajas}

Variedad de metodologías de enseñazaaprendizaje: aprendizaje cooperativo; aprendizaje basado en casos reales; redacción de informes, etc.

Variedad de procedimiento de evaluación inscritos en actividades prácticas

Rol activo del alumnado

Aprender haciendo

\section{Inconvenientes}

Profesorado Asociado poco implicado en la implementación de las vías de evaluación de dichas competencias, en ocasiones

Coordinación deficiente entre las asignaturas que evaluan la misma Competencia Transversal

Escasez de laboratorios informáticos disponibles, lo que disminuye la eficiencia de la enseñanzaaprendizaje.

Fuente: elaboración propia.

\footnotetext{
${ }^{2}$ En este sentido, véase el trabajo de Sáez y Ruiz (2012: 374 y 376), donde se señala que el proceso de "aprender haciendo" permite desarrollar competencias cognitivas y metacognitivas, que potencian los recursos de comprensión del alumnado, esenciales en su futuro profesional.
} 


\section{Conclusiones}

La Competencia Transversal "Comprensión e Integración” está en el corazón de la docencia al formar parte de los objetivos que se persiguen. No obstante, es necesaria la coordinación entre los profesores que están evaluando la misma Competencia para poder verificar si de forma continuada los procedimientos de evaluación están detectando la adquisición de la competencia en los dos niveles de dominio que manejamos en el Grado de Gestión de Administración Pública.

La variedad de actividades formativas y de procedimientos de evaluación, confirman que la Competencia se adquiere y que se puede determinar su adquisición por el estudiante de forma correcta. Esto se contrasta con los resultados de cada asignatura, especialmente el rendimiento académcio obtenido en cada una de ellas, muy por encima del $85 \%$ de media, y que evidencia que el alumno tiene la competencia. Se considera que disponer de un criterio homogéneo de evaluación permite aclarar a los alumnos los resultados obtenidos, y no generar dudas y posibles reclamaciones.

De este modo el valor añadido para este Grado se incrementa de forma sustancial al poder ser contratastable el nivel de dominio de las Competencias Transversales al realizarse su evaluación de forma sistemática.

\section{Referencias}

ARROYO-CAÑADA, F-J. (2019). "La evaluación por competencias en estudios empresariales". Journal of Management and Business Education, vol. 2 (1), p. 1-7

GONCZY, A. (2001). Análisis de las tendencias internacionales y de los avances en educación y capacitación laboral basadas en normas de competencias. En A. Argüelles \&A. Gonczy, Educación y capacitación basadas en normas de competencias (p. 38-40). México :Limusa.

SÁEZ LÓPEZ, J. L. y RUIZ RUIZ, J. Ma . (2012). "Metodología didáctica y tecnología educativa en el desarrollo de las competencias cognitivas: aplicación en contextos universitarios". Profesorado. Revista de Currículum y formación del profesorado, vol. 16 (3), p. 373-391 <https://www.ugr.es/ recfpro/rev163COL9.pdf> [Consulta: 06 de mayo de 2019].

UPV (2015). Proyecto Competencias transversales. Universitat Politècnica de València $<$ https://www.upv.es/entidades/ICE/info/Proyecto_Institucional_CT.pdf $>$ [Consulta: 06 de mayo de 2019]. 